\title{
Temperature-Dependent H/D Exchange of Compact and Elongated Cytochrome $c$ Ions in the Gas Phase
}

\author{
Stephen J. Valentine \\ Beyond Genomics, Inc., Waltham, Massachusetts, USA \\ David E. Clemmer \\ Department of Chemistry, Indiana University, Bloomington, Indiana, USA
}

\begin{abstract}
Isotopic exchange reactions of compact and elongated conformations of gaseous cytochrome $c$ ions ( +5 and +9 states) with $\mathrm{D}_{2} \mathrm{O}$ have been measured as a function of temperature (from 300 to $\sim 440 \mathrm{~K}$ ) using ion mobility techniques. Rate constants for those sites that exchange at high temperatures $(>400 \mathrm{~K})$ are about an order of magnitude smaller than rate constants for sites that exchange at $300 \mathrm{~K}$. Although the exchange rates decrease, the maximum exchange levels for rapidly exchanging sites increase with temperature. At $300 \mathrm{~K}$, exchange levels of $53 \pm 3$ and $63 \pm 3$ are measured for the compact and elongated states, respectively. From 300 to $335 \mathrm{~K}$, the exchange levels increase slightly to $\sim 60$ to 70 hydrogens. Above $335 \mathrm{~K}$, the levels increase to a value of $\sim 200$ for the +5 state and $\sim 190$ for the +9 state, near the maximum possible levels, 200 and 204 for these respective charge states. Molecular dynamics simulations have been carried out on structures having calculated cross sections that are near the experimental values in order to explore the exchange process. Overall, it appears that charge site and exchange site proximities are important factors in the exchange profiles for the elongated +9 ion and the compact +5 ion. (J Am Soc Mass Spectrom 2002, 13, 506-517) (c) 2002 American Society for Mass Spectrometry
\end{abstract}

I is well known that the structures of proteins are influenced by environmental factors such as solution composition, $\mathrm{pH}$, and temperature. However, the degree to which environment and intrinsic properties influence structure is not well understood. Structural domains such as $\alpha$-helices, which are stable in a wide range of environments, were predicted theoretically without considering effects of solvation [1]. More subtle features, such as tertiary structure in different environments, are difficult to predict. Recently, efforts have been made to gather information about the degree to which solvent-free protein conformations resemble their native solution structures [2]. Several studies of lyophilized protein powders have shown that removal of solvent can cause structural changes [3], and the extent of differences between lyophilized and solution states is an active area [4]. New ion sources [5,6] make it possible to isolate biomolecules in the gas phase as ions, and a number of mass spectrometry (MS) based

Published online March 27, 2002

Submitted to J. Am. Soc. Mass Spectrom in honor of Professor Armentrout's Biemann Award on December 31, 2001.

Address reprint requests to Dr. David E. Clemmer, Department of Chemistry, Indiana University, Bloomington, Indiana 47405, USA. E-mail: clemmer@indiana.edu strategies for examining solvent-free conformations are being developed [7-32]. The new information from these technologies is beginning to be evaluated by theoretical methods $[33,34,35]$. There is now substantial evidence that, under some experimental conditions, anhydrous protein and peptide ions may retain elements of solution conformation.

The conformations of cytochrome $c$ ions have been examined previously by a number of MS techniques [8, 28]. Ion mobility (for reviews of ion mobility studies see [30]) and isotopic exchange studies [7-10, 11, 12] provide information about the overall shape and number of accessible exchange sites, respectively. These studies indicate the presence of multiple conformations within individual charge states, and have shown structural transitions in the gas phase resembling solution-phase folding and unfolding processes [9, 28b, 32]. Ion scattering [25, 26] and ion mobility studies [27-30] show that high charge states of the protein adopt elongated conformations, presumably to reduce high coulomb repulsion energies [25, 28]. It is somewhat surprising that isotopic exchange levels as a function of charge state do not show a corresponding increase $[8,9,11,12]$.

Our initial interest in this system arose from early work by McLafferty and coworkers, who used fourier 


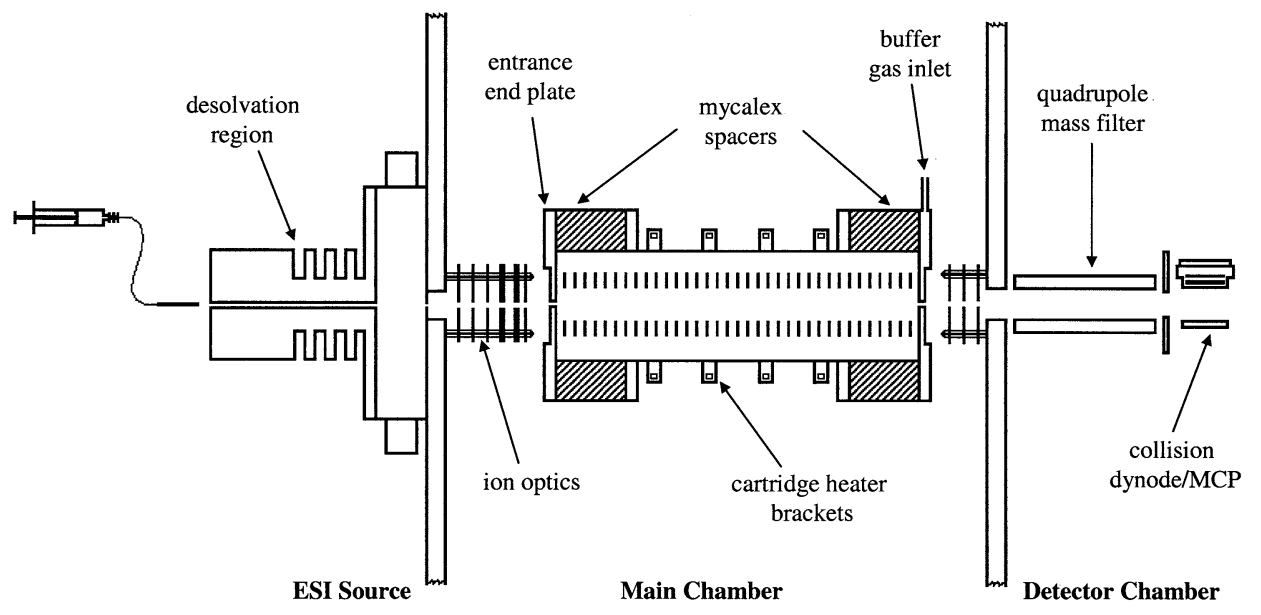

Figure 1. Schematic diagram of the experimental apparatus.

transform (FT) MS methods to expose ions to low concentrations of $\mathrm{D}_{2} \mathrm{O}$ for long time periods (up to 30 minutes) [8]. In an earlier study, using a combined ion mobility/isotopic exchange approach, we reported limited exchange for both the compact and extended conformers relative to the FTMS results. At $300 \mathrm{~K}$, a maximum exchange level of $\sim 46$ sites (out of a possible $196+\mathrm{nH}$, where $\mathrm{n}$ is the number of protons added during electrospray) was reported for compact conformers (observed for the +8 through +10 charge states); the most elongated conformers observed for higher-charge state ions $(+8$ to +18$)$ exchanged more, $63 \pm 2$, however, still far below exchange levels observed by FTMS [11]. ${ }^{+}$

In this paper we report isotopic exchange studies of compact and elongated structures over a temperature range of 300 to $440 \mathrm{~K}$. There are three primary experimental findings: 1) rate constants for saturation of those sites that exchange at $300 \mathrm{~K}$ are about an order of magnitude larger than rate constants for saturation of sites that exchange at $400 \mathrm{~K} ; 2$ ) the maximum exchange levels for both conformers increase with temperature to near the maximum possible levels; and, 3) at high temperatures $(>380 \mathrm{~K})$ the exchange level of compact conformers exceeds the level of the elongated structure. Molecular dynamics simulations have been carried out (at several temperatures) in order to understand what factors influence the exchange process. As the simulation temperature is increased both the compact and elongated conformers sample more conformations. However, the retention of a compact structure for the +5 allows more exchangeable sites to come into close proximity with protonation sites; whereas, regions of exchangeable sites on the +9 ion do not appear to have access to the protonation sites because of the elongated nature (and rigidity) of this state.

The present work is also closely related to tempera-

${ }^{+}$The total number of exchangeable sites for equine cytochrome $c$ in solution is 198. Recent work (e.g., Refs. [12] and [43]) indicates that in the electrosprayed ions, two carboxyl groups on the heme are deprotonated. ture-dependent cross section measurements for the +5 to +7 states carried out by Jarrold and coworkers [36, 37]. They find that the +5 state is very stable, retaining a compact conformation even at extreme temperatures $(\sim 570 \mathrm{~K})$. Compact conformers of higher charge states unfolded at higher temperatures.

\section{Experimental}

\section{General}

A schematic diagram of our experimental apparatus is shown in Figure 1. Ion mobility techniques [30] and our basic experimental procedures [11, 22] are described elsewhere. Only a brief description is given here. Multiply charged cytochrome $c$ ions are formed by electrospraying a $8.0 \times 10^{-5} \mathrm{M}$ cytochrome $c$ (horse heart, Sigma $>99 \%$ ) solution in pure $\mathrm{H}_{2} \mathrm{O}$. Electrosprayed droplets are formed at atmosphere and enter a variabletemperature, differentially-pumped desolvation region through a $0.1 \mathrm{~cm}$ diameter entrance orifice. Protein ions exit the source through another $0.1 \mathrm{~cm}$ diameter orifice into a high vacuum region $\left(10^{-4}\right.$ to $10^{-5}$ torr) where they are focused into a low-energy ion beam, and injected into the drift tube containing $\sim 2.0$ torr of $\mathrm{He}$ buffer gas through a $0.08 \mathrm{~cm}$ diameter aperture. Ions drift through the buffer gas under the influence of a weak uniform electric field (from 1.42 to $14.2 \mathrm{Vcm}^{-1}$ ) provided by equally-spaced electrostatic lenses and a small fraction exit through another $0.08 \mathrm{~cm}$ diameter aperture where they are focused into a quadrupole mass filter. The quadrupole can be fixed to examine individual charge states or scanned to record a mass spectrum.

\section{Measurement of H/D Exchange Levels and Kinetics}

The number of hydrogens that exchange is obtained by comparing the mass spectra recorded with and without 
$\mathrm{D}_{2} \mathrm{O}$ inside the drift tube and is given by the $m / z$ shift in the centers of the peaks multiplied by the charge state. Peak centers can be determined to within 0.3 to $0.5 \mathrm{~m} / \mathrm{z}$; the higher uncertainties are associated with data where exchange is incomplete. Under conditions where the maximum exchange level (for a particular temperature) is reached, uncertainties for our exchange levels are \pm 3 or \pm 6 for the +5 or +9 charge states, respectively.

Rate constants associated with the average rate of $\mathrm{H} / \mathrm{D}$ exchange for the ensemble of sites can be obtained from semilog plots of the number of fast-reacting hydrogens that remain against the solvent pressure multiplied by the drift time. The partial pressures of $\mathrm{He}$ and $\mathrm{D}_{2} \mathrm{O}$ are measured with a capacitance manometer by a difference method described previously [11]. Between each measurement, the partial pressures of the gasses are checked by removing one of the reagent gasses. When either gas is removed, the pressure quickly equilibrates to the pressure of the remaining gas. Drift times are measured at each solvent pressure as described below and vary from $\sim 1.5$ to $30 \mathrm{~ms}$. The drift time can be controlled by changing the drift field.

\section{Measurements of Maximum Exchange Levels as a Function of Temperature}

Below we report maximum exchange levels as a function of temperature. Maximum exchange values were determined from mass spectra recorded when the buffer gas is doped with $\sim 0.7$ torr of $\mathrm{D}_{2} \mathrm{O}$. Low drift fields of $\leq 3 \mathrm{Vcm}^{-1}$ were used such that typical residence times of the +5 and +9 ions inside the drift tube were between $\sim 20$ to $30 \mathrm{~ms}$. As shown below, these conditions were found to be sufficient to saturate what we refer to as rapidly exchanging sites at all of the temperatures we have studied.

\section{Ion Temperature}

Once injected into the drift tube, protein ions experience $\sim 10^{5}$ to $10^{6}$ collisions/cm with the buffer gas and ions rapidly equilibrate to the temperature of the buffer gas. The temperature of the buffer gas is varied by heating the drift tube body with eight electric cartridge heaters mounted in heater brackets as shown in Figure 1. The temperature that is reported is an average of values recorded from three thermocouples mounted at the center and on the entrance and exit flanges. In a typical experiment, the drift tube body is heated and then heaters are turned off to allow the mycalex spacers and endcaps to equilibrate to the temperature of the drift tube (Figure 1). After several minutes of equilibration time, we begin collecting data. Near room temperature, there is little ambiguity in the temperature. For example typical average values and ranges are 295.0 $\pm 0.1,325 \pm$ $2,356 \pm 3$ and $379 \pm 6 \mathrm{~K}$. At higher temperatures, the range increases such that average values and ranges of $392 \pm 8,406 \pm 13,450 \pm 20 \mathrm{~K}$, are measured. In separate experiments we have inserted thermocouples inside the drift tube and recorded gas temperatures while monitoring the temperature of the drift tube body and endcaps. These experiments suggest that a temperature gradient along the drift axis (similar to that on the surface of the drift tube) may be present; this gradient is most apparent at high temperatures.

\section{Conformer Characterization}

The mobilities of ions depend largely on their shapes and charge state; for a given charge state, compact conformations have higher mobilities than more diffuse ones. Ion mobility distributions were recorded by injecting short pulses (30 to $50 \mu \mathrm{s}$ ) of ions into the drift tube and recording the arrival time distribution at the detector as described previously $[11,30]$. The arrival time is a composite of the ions' drift time $\left(t_{D}\right)$ through the buffer gas and flight time through remaining portions of the instrument. In these experiments, flight times (recorded without the buffer gas) ranged from 190 to $270 \mu \mathrm{s}$. For temperature-dependent data, it is useful to display ion mobility distributions as cross section distributions in order to normalize for differences in ion diffusion at different temperatures. This can be done by using the relation [38]

$$
\begin{aligned}
\Omega= & \frac{(18 \pi)^{1 / 2}}{16} \frac{z e}{\left(k_{B} T\right)^{1 / 2}}\left[\frac{1}{m_{I}}+\frac{1}{m_{B}}\right]^{1 / 2} \\
& \frac{t_{D} E}{L} \frac{760}{P} \frac{T}{273.2} \frac{1}{N}
\end{aligned}
$$

where $z, e$, and $k_{B}$ are the charge state, electron charge and Boltzmann's constant, respectively; the $E, L, P$, and $T$ variables correspond to the experimental parameters electric field, length of drift region, and buffer gas pressure and temperature, respectively. The variable $N$ is the neutral number density, and $m_{I}$, and $m_{B}$, are the masses of the ion and buffer gas, respectively. All of the experimental parameters $\left(E, L, P, T\right.$ and $\left.t_{D}\right)$ can be precisely measured. At $\sim 300 \mathrm{~K}$, the reproducibility of the measured cross sections is excellent; different measurements agree to within $1 \%$ of one another (relative uncertainty) and within $2 \%$ of values reported previously [28a]. Cross sections determined at high temperatures for the +5 and +7 states are also in good agreement (within a few percent) with values measured previously [36].

\section{Influence of Solvent Vapor on Conformation}

An additional experimental consideration in these studies is the possibility that water vapor can induce changes in the conformation of the gas phase ions. To test for this we recorded ion mobility distributions as a 
function of water vapor pressure (both $\mathrm{H}_{2} \mathrm{O}$ and $\mathrm{D}_{2} \mathrm{O}$ ) as we described in detail previously [11]. We observe no evidence for adduct formation under any of the conditions used in these experiments. ${ }^{\ddagger}$ As the partial pressure of $\mathrm{D}_{2} \mathrm{O}$ is increased, the drift times of each conformation systematically increases, due to the decreased mobility of the ion through $\mathrm{D}_{2} \mathrm{O}$ compared with He. However, there is no evidence that structural transitions occur because of interactions between the cytochrome $c$ ions with the water vapor as observed in the ion-mobility distributions [11]. It is also possible that ions undergo proton transfer reactions while they are drifting through the tube in the presence of $\mathrm{D}_{2} \mathrm{O}$. We cannot rule out the possibility that a small fraction of very high-charge state ions may undergo proton transfer reactions immediately upon entering the drift tube. However, mass spectra recorded with and without the deuterated solvent are similar, and show no evidence for charge transfer processes. By recording ion mobility distributions at varying drift voltages, we can unambiguously state that the +5 and +9 ions are not involved in proton transfer reactions as they drift through the drift tube; this is consistent with the measured gas-phase basicities for these charge states of cytochrome $c$ [20].

\section{Molecular Dynamics Simulations}

Molecular dynamics simulations have been carried out using the Discover 3.0 software with the ESFF force field. ${ }^{\S}$ We use the compact NMR structure (the native solution state) as the initial structure for the +5 charge state [36]. The initial structure used for the +9 ion was generated by retaining the $\alpha$-helical regions found in solution as well as forming several short helical sections from regions of the protein primary sequence containing a higher percentage of residues with a greater propensity to form helices. These helical sections include residues 19-27, 32-39, and 79-89. In the remainder of the molecule, $\varphi, \psi$, and $\omega$ bond angles were set for a linear backbone. This conformer is referred to as the linear/helix structure and has a calculated cross section of $2150 \AA^{2}$ [28a]. The calculated cross section of the NMR structure is $1165 \AA^{2}$. The initial structures for the molecular dynamics simulations are shown in Figure 2. Molecular dynamics simulations of both structures were carried out in $1 \mathrm{fs}$ timestep increments at 300, 400, 500,600 , and $1000 \mathrm{~K}$ for $0.5 \mathrm{~ns}$, a process that required

${ }^{\ddagger}$ Extraction voltages in pressure interface regions can effectively decluster protein-water adducts. We have tested for this by systematically varying the extraction potential between the drift tube and quadrupole sections of our instrument and find no evidence for protein-water adducts. The absence of adduct formation is consistent with recent measurements of the binding enthalpies for the first waters to associate with cytochrome $c$, which were measured at low temperatures. See Ref. [28d].

\$These models were created using Insight II, BIOSYM/MSI, San Diego, CA (1995).
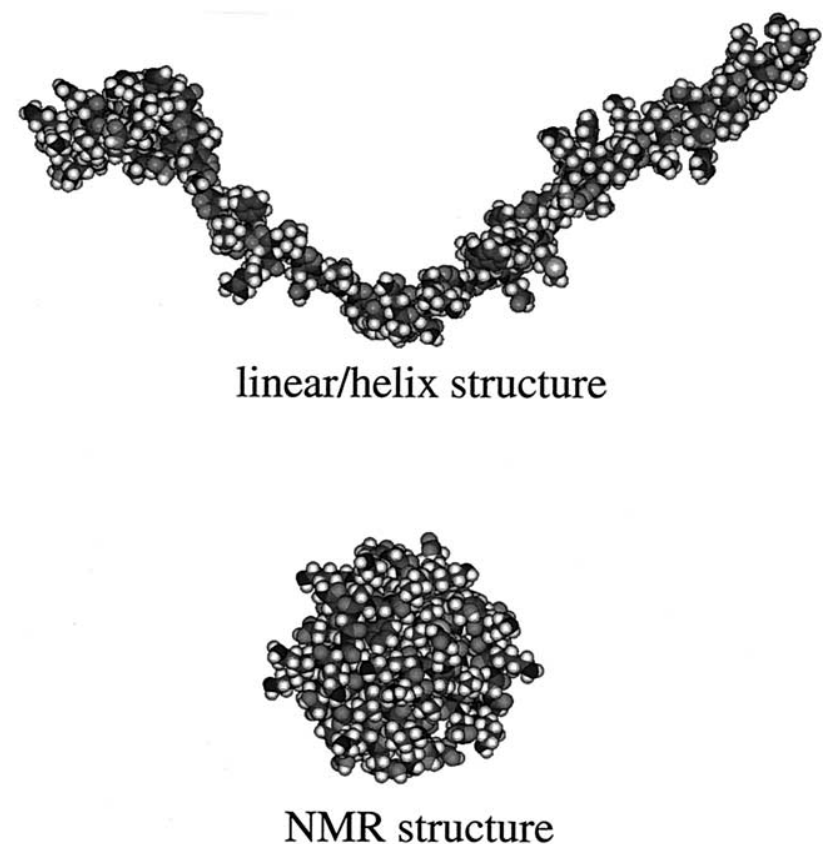

Figure 2. Space-filled models of the initial structures used for the molecular dynamics studies. The linear/helix structure and the NMR structure were used to represent the +9 and +5 charge states, respectively.

$\sim 6$ months using an Indigo 2 silicon graphics computer with a R10000 processor.

\section{Charge Placement in Simulated Structures}

An important issue that arises in multiply charged systems is the placement of charges. As discussed previously [36, 39], it is impossible to calculate the total ion energy for all possible charge site assignments for each structure [20a, 36]. Here, we assume formal charges of +3 on the heme iron atom and -1 on each heme carboxylate group [12, 40] Added protons from the electrospray process are confined to basic Arg, His, or Lys residues. The total number $(n)$ possible charge site configurations for a protein is given by

$$
n=\frac{x !}{y !(x-y) !}
$$

where $x$ and $y$ correspond with the number of basic sites and the number of protons for charge placement. For $\mathrm{N}$-terminal acetylated cytochrome $c$, the total number of ways to place 4 or 8 protons (for the +5 and +9 charge states, respectively) ${ }^{+\dagger}$ along 24 basic residues is $\sim 1.1 \times$ $10^{4}$ and $7.4 \times 10^{5}$, respectively. Coulomb energies of all possible charge site configurations were calculated for the energy-minimized compact and elongated structures by using the expression

${ }^{++}$Because the heme group has a net charge of $+1, \mathrm{n}$ is equal to 4 and 8 for the +5 and +9 charge states, respectively. 


$$
\text { C.E. }=\sum_{i=1}^{n-1} \sum_{j=i+1}^{n} \frac{q^{2}}{4 \pi \varepsilon_{0} \varepsilon r_{i j}}
$$

where $\varepsilon_{0}$ is the permittivity of free space, and $\varepsilon$ is the dielectric constant (1.0 for these in vacuo simulations), and $r_{i, j}$ is the distance between charges assigned to the $i$ and $j$ locations. The lowest Coulomb energy configuration found by this process was utilized in the molecular dynamics simulations. After charges have been placed at basic sites, the structures were again energy minimized prior to initiating molecular dynamics simulations.

\section{Assessing the Number of Exchangeable Hydrogens on a Calculated Structure}

Simulated structures provide an opportunity to address issues regarding the exchange process; specifically we address how temperature influences the number of exchangeable sites that become available as the protein samples different conformations. H/D exchange of small ions with a variety of reagents has received considerable attention [16, 41, 42]. Mechanisms associated with larger systems are less clear; however, several models have been proposed [16, 19] (For a recent review of ion-molecule reactions as probes of gas-phase conformation also see [13]). Beauchamp and coworkers have previously proposed that $H / D$ exchange between small peptide oligomers and $\mathrm{D}_{2} \mathrm{O}$ proceeds via a relay mechanism involving complexes where the $\mathrm{D}_{2} \mathrm{O}$ interacts through hydrogen bonds at two sites on the peptide [16]. H/D exchange of specific backbone and sidechain sites would require their proximity to the less basic site of the reaction complex [16, 19]. As a first approximation for the modeling studies, we assume that the exchange hydrogens must necessarily be positioned near a charge site during the dynamics simulation an approach that is similar to a model discussed by Wyttenbach and Bowers [19]. For each conformer, one hundred archived structures (energy minimized and saved each 5 ps of the simulation) were analyzed for distances between exchange hydrogens and charge sites. Exchange hydrogens within specified lengths of the charge sites were designated as "accessible" for exchange. Figure 3 shows a $\mathrm{D}_{2} \mathrm{O}$ molecule interacting with a portion of the cytochrome $c$ sequence that includes the charged Lys 72 residue. A circle, centered at the charge site, is used to represent the volume through which exchangeable sites would be considered to be accessible. Here we assume that sites within $5 \AA$ of the protonation site are accessible and those at greater distances do not exchange. The total number of exchange sites is taken as the total number of sites that fall within the exchange volume at any time interval during the dynamics simulation. This effectively assumes that a $\mathrm{D}_{2} \mathrm{O}$ molecule is always present for exchange.

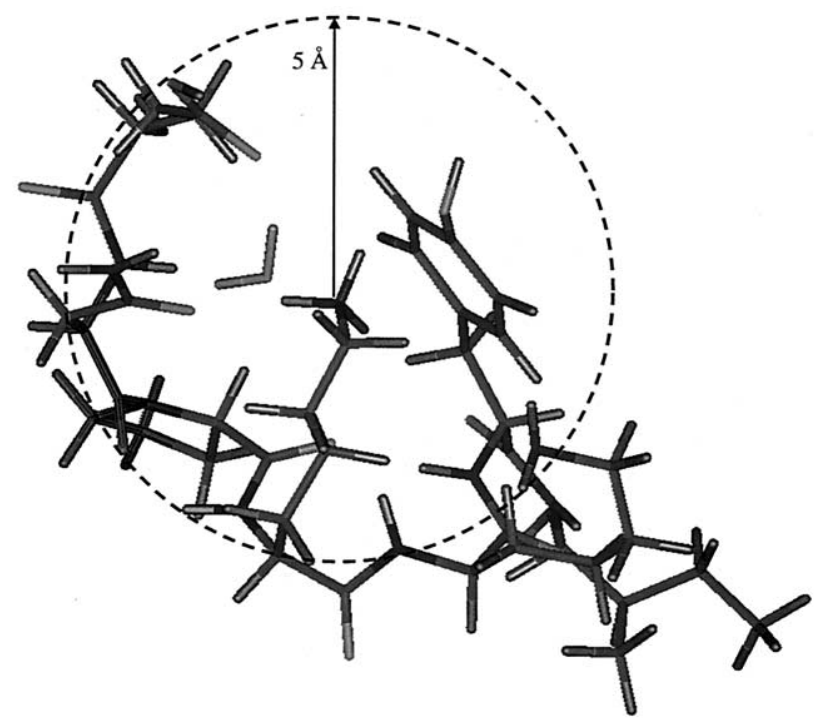

Figure 3. Stick representation of a six-residue portion of the cytochrome $c$ sequence that contains the charged Lys 72 residue interacting with a $\mathrm{D}_{2} \mathrm{O}$ molecule. This portion of the peptide was taken from a structure generated by modeling (chosen randomly). The dashed-line circle represents an accessible H/D exchange region having a radius that extends $5 \AA$ from the protonation site.

\section{Results and Discussion}

Temperature-dependent Cross Sections for the +5 and +9 Ions

Figure 4 shows cross section distributions for the +5 and +9 states that were recorded at temperatures of 300 and $400 \mathrm{~K}$. The conformations for the +5 ions have cross sections centered around $1242 \AA^{2}$ at $300 \mathrm{~K}$, and $1210 \AA^{2}$ at $400 \mathrm{~K}$. The peaks are too broad to be accounted for by a single structure. Thus, either multiple conformers with similar cross sections are present, or conformers are interconverting between different compact forms (on the timescale of the experiment) as they travel through the drift tube. Figure 4 also shows the location of the cross section that has been calculated previously for coordinates for the native solution structure of cytochrome $c\left(1080 \AA^{2}\right)$ calculated from the projection approximation method [28a]. These comparisons, and results obtained at other temperatures, indicate that the +5 state is extremely compact under all of the conditions of our experiments. The measured cross section of the +9 state is $2180 \AA^{2}$ at $300 \mathrm{~K}$, and $2155 \AA^{2}$ at $400 \mathrm{~K}$, values that are $\sim 75 \%$ larger than the cross sections measured for the +5 state. We have calculated projection and EHSS cross sections [28a, 43] for a wide range of randomly chosen cytochrome $c$ conformations that were created by molecular modeling. ${ }^{\S}$ Comparison of these values with our experimental data show that only conformers with virtually no tertiary structure have cross sections that are comparable to the +9 data; the +9 state must be a highly-open form (Figure 2). The

\$These models were created using Insight II, BIOSYM/MSI, San Diego, CA (1995). 


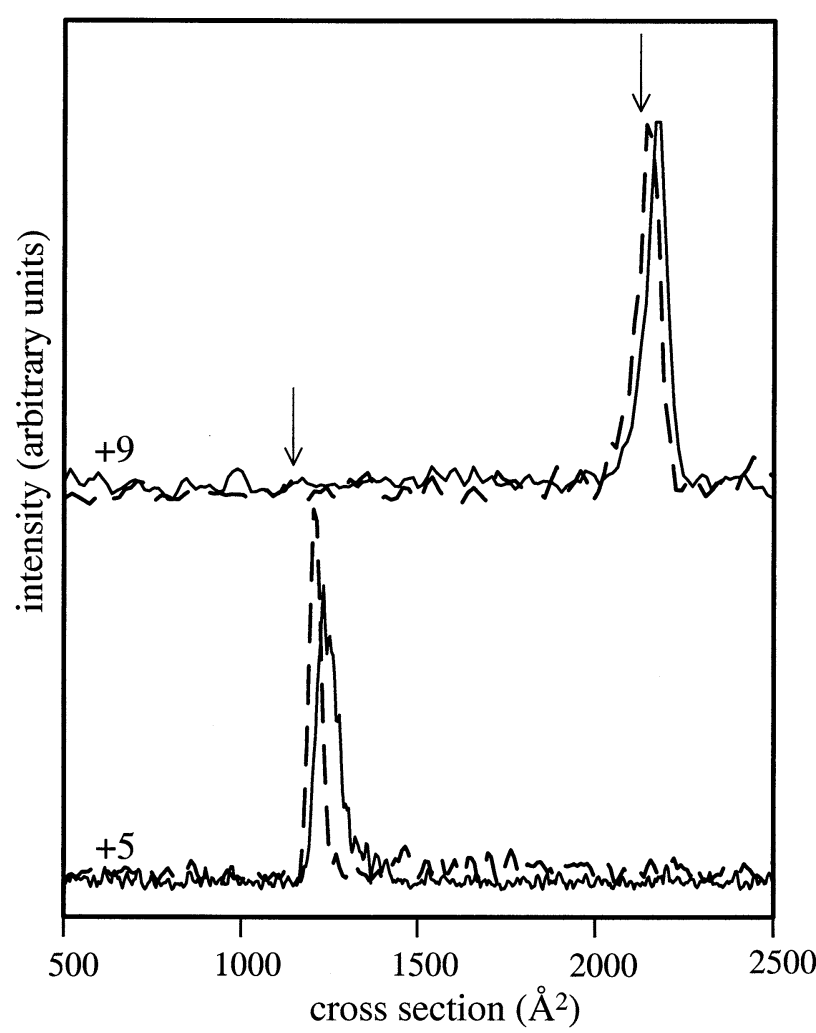

Figure 4. Cross section distributions (see text) recorded for the +5 and +9 states of cytochrome $c$ at buffer gas temperatures of 300 $\mathrm{K}$ (solid lines) and $\sim 400 \mathrm{~K}$ (dashed lines). The data were recorded using injection energies of 2250 and $4050 \mathrm{eV}$ for the +5 and +9 states, respectively. The arrows show cross sections calculated for the compact NMR conformation and the elongated linear/helix conformer using the projection approximation method.

cross section that we measure is near an open conformer having an entirely $\alpha$-helical structure [28c] This type of structure is expected to become stable in the gas phase near the +9 and higher charge states because there are a large number of hydrogen bonds (associated with the helix) and the elongated structure reduces coulombic repulsion [28c]. The +9 experimental cross section is also near the value of $2149 \AA^{2}$ for the linear/ helix structure that is used as the initial structure in the molecular dynamics simulations.

The cross section distributions of the +5 and +9 states vary only slightly with temperature, indicating that there are no large changes in overall shapes of these ions ${ }^{|l| \mid}$. These results indicate that a compact conformer is most stable for the +5 state (as observed previously) $[28,36]$ and an elongated form is most stable for the +9 state. Although we do not present H/D exchange studies for the +6 to +8 states here, it is noteworthy that experimental results show that thermally-induced structural transitions, similar to those reported previ-

III Cross sections as a function of temperature vary slightly because of differences in relative contributions of hard-sphere and attractive ioninduced dipole and van der Waals contributions to the collision cross section as a function of temperature. These effects have been discussed previously. See for example Ref. [44].
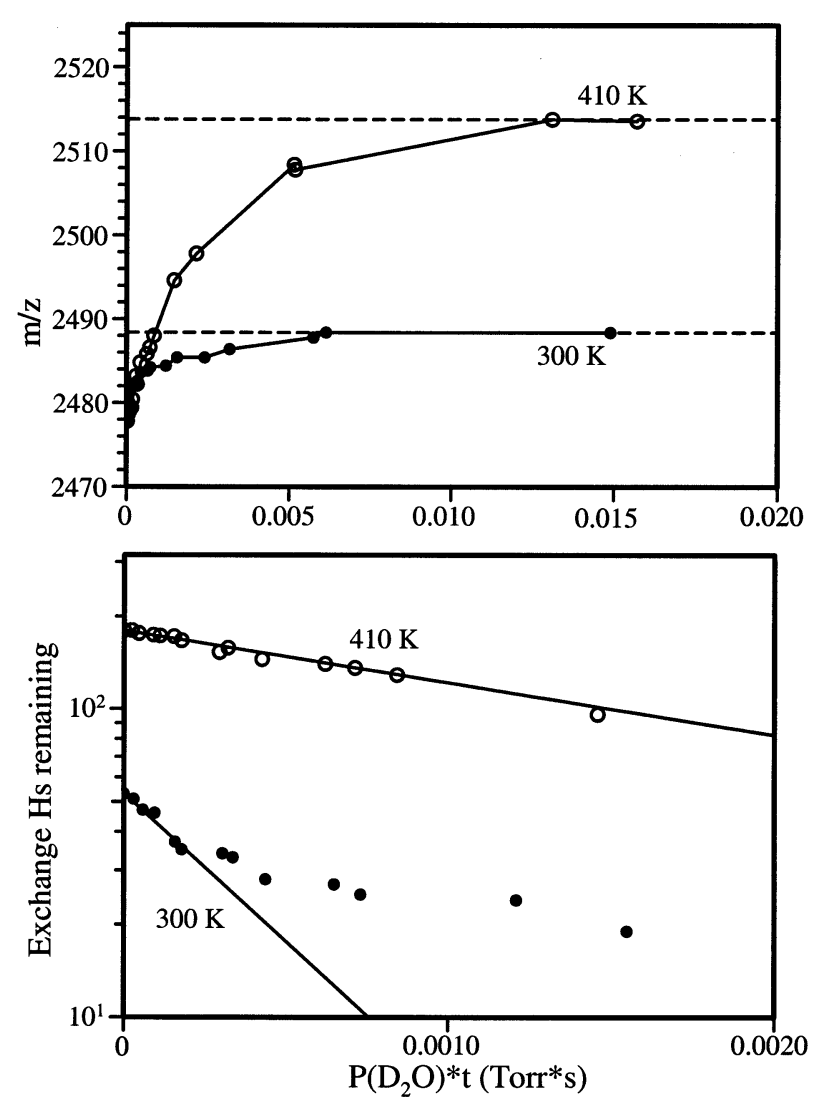

Figure 5. The top graph shows $\mathrm{H} / \mathrm{D}$ exchange saturation profiles measured from the $\mathrm{m} / \mathrm{z}$ shift plotted against the $\mathrm{D}_{2} \mathrm{O}$ pressure multiplied by the residence times of the ions in the drift tube $\left[\mathrm{P}\left(\mathrm{D}_{2} \mathrm{O}\right) \times t\right]$ at buffer gas temperatures of $\sim 300$ and $\sim 410 \mathrm{~K}$ for the +5 charge state of cytochrome $c$. The maximum exchange level is found from the maximum $\mathrm{m} / \mathrm{z}$ shift multiplied by the charge state and is shown as dashed lines for each temperature. The bottom graph shows kinetic plots of the number of exchangeable hydrogens that remain as a function of $\mathrm{P}\left(\mathrm{D}_{2} \mathrm{O}\right) \times t$ for the +5 state at these temperatures. These plots are obtained by subtracting exchange levels at specific values of P.t from the maximum exchange level observed at a specific temperature. For the data shown, the maximum exchange levels at 300 and $410 \mathrm{~K}$ are 53 and 180 sites, respectively. This type of plot is analogous to the kinetic plots reported previously, see, for example, Ref. [8].

ously for the +7 state [30e, 36], are observed over this temperature range.

\section{H/D Exchange Kinetics}

Figure 5 shows the measured $m / z$ values from the centers of peaks in the mass spectra plotted against the deuterated solvent pressure multiplied by the drift time $\left[\mathrm{P}\left(\mathrm{D}_{2} \mathrm{O}\right) \cdot \mathrm{t}\right]$ for the +5 state at buffer gas temperatures of 300 and $410 \mathrm{~K}$. In virtually all of our data, all fastreacting hydrogens appear to have exchanged by $\sim 0.01$ torr.s. From the $\mathrm{m} / \mathrm{z}$ maxima, we determine exchange levels for fast reacting sites of 53 at $300 \mathrm{~K}$, and 180 at 410 $\mathrm{K}$. Data for the +9 state are similar, and the exchange level measured at $300 \mathrm{~K}$ (63 hydrogens) is in good agreement with our previous results $(64 \pm 3)$ [11].

Figure 5 also shows typical kinetic plots of our data 
Table 1. Temperature dependent $H / D$ exchange kinetics for the +5 and +9 charge states ${ }^{a}$

\begin{tabular}{lcccc}
\hline$Z$ & $300 \mathrm{~K}$ & $350 \mathrm{~K}$ & $373 \mathrm{~K}$ & $410 \mathrm{~K}$ \\
\hline \hline+5 & $9.7^{\mathrm{b}}(53)$ & $4.0(75)$ & $3.7(95)$ & $1.2(180)$ \\
& $2.6^{\mathrm{c}}$ & & & \\
+9 & $51.8^{\mathrm{b}}(63)$ & $5.8(83)$ & $7.5(96)$ & $4.2(150)$ \\
& $11.3^{\mathrm{c}}$ & & & \\
\hline
\end{tabular}

all rate constants are reported as $\mathrm{k}_{\mathrm{H} / \mathrm{D}} \times 10^{14} \mathrm{~cm}^{3} \cdot \mathrm{s}^{-1} \cdot$ molecule $^{-1}$ and have estimated uncertainties of $\pm 50 \%$. The maximum $H / D$ values that are determined from the mass spectra are given in parentheses. Note that these values correspond to the kinetics associated with saturating those sites that can react during our experiment. Comparison of these results is somewhat ambiguous since different sites may become accessible at different temperatures, even within the same structural type (compact or elongated).

${ }^{b}$ Multiple features were present in the semilog plot. These rate constants were estimated by fitting the fastest reacting hydrogens.

cMultiple features were present in the semilog plot. These rate constants were estimated by fitting the more slowly reacting hydrogens.

that can be used to estimate average rate constants for $\mathrm{H} / \mathrm{D}$ exchange; the rate constants are reported for several temperatures in Table 1 . Values range from $\sim 1$ to $50 \times 10^{-14} \mathrm{~cm}^{3} \cdot \mathrm{s}^{-1} \cdot$ molecule ${ }^{-1}$ and indicate that these reactions are slow at all temperatures. For the compact $(+5)$ conformation, a collision rate constant is calculated to be $\sim 2.2 \times 10^{-8} \mathrm{~cm}^{3} \cdot \mathrm{s}^{-1} \cdot \mathrm{s}$ molecule ${ }^{-1}$. Using this rate constant and an exchange rate constant of $9.7 \times 10^{-14} \mathrm{~cm}^{3} \cdot \mathrm{s}^{-1} \cdot$ molecule $^{-1}$ (Table 1 ), approximately $2.3 \times 10^{5}$ collisions would be required for deuteration of the 53 sites at $300 \mathrm{~K}$. This corresponds to $\sim 4.3 \times 10^{3}$ collisions, on average, for each exchange event. These values indicate that reactions are not facile for either charge state over the temperature range studied [16].

From a few studies at higher $\mathrm{D}_{2} \mathrm{O}$ pressures and longer exposure times in the drift tube (up to $\sim 1$ s) we estimate that rate constants for those sites that do not exchange in our experiments must be less than $1 \times$ $10^{-15} \mathrm{~cm}^{3} \cdot \mathrm{s}^{-1} \cdot$ molecule $^{-1}$. It is also notable that at $300 \mathrm{~K}$, the data are generally linear over a range of $\mathrm{P}\left(\mathrm{D}_{2} \mathrm{O}\right) \cdot \mathrm{t}$ values up to $\sim 0.0003$ torr.s. Above these values, the deviation from linearity indicates that multiple exchange processes with different rate constants are present; a smaller $\mathrm{x}$-axis range is used in the kinetic plot to more clearly distinguish these processes. This nonlinearity is also observed in kinetics experiments performed at $300 \mathrm{~K}$ for the +9 charge state. We have analyzed the different components of the room-temperature data as two processes and estimate that rate constants for the faster reacting component are about a factor of five times larger than those of the more slowly reacting component (Table 1 ). It is likely that more than two components contribute to the nonlinearity. Although exchange hydrogens react with different rates, Figure 5 suggests that $\sim 20$ react at faster rates while $\sim 33$ react at slower rates. As analyzed, the linearity of the slower exchange process at $300 \mathrm{~K}$ and the exchange process at high temperatures continues to P.t values of $\sim 0.005$ torr.s, near complete saturation of all exchange sites.
Variability in exchange rate constants should arise because of differences in the intrinsic exchange rates of diverse sites along the protein; variations may also be due to the presence of multiple conformations (with similar cross sections) or structural changes that occur as the protein drifts through the instrument. To test whether or not structural changes occur as ions drift through the instrument, we recorded H/D exchange profiles at different drift voltages (which provide an order of magnitude variation in the exposure time of ions to deuterated solvents). The kinetic plots obtained in these studies are identical within experimental uncertainty to those in Figure 5. This indicates that these conformations either are stable and do not interconvert over the $\sim 1$ to $30 \mathrm{~ms}$ timescales of these experiments; or, if interconversion occurs, it must do so rapidly compared with our experimental timescales. The present results are consistent with those found in FTICR experiments where structures do not appear to interconvert over very long time periods (several minutes) $[8,9,12]$.

Although the maximum exchange levels increase with temperature, the rapid processes observed at low temperatures are squelched as the temperature is increased and the overall rate constant for saturating the number of sites that can exchange decreases. At $\sim 410 \mathrm{~K}$, exchange rate constants of $1.2 \times 10^{-14} \mathrm{~cm}^{3} \cdot \mathrm{s}^{-1} \cdot$ molecule $^{-1}$ for the +5 and $4.2 \times 10^{-14} \mathrm{~cm}^{3} \cdot \mathrm{s}^{-1} \cdot$ molecule $^{-1}$ for the +9 states are factors of $\sim 10$ times smaller than the rapid processes observed at $300 \mathrm{~K}\left(9.7\right.$ and $51.8 \times 10^{-14}$ $\mathrm{cm}^{3} \cdot \mathrm{s}^{-1} \cdot$ molecule ${ }^{-1}$ for these respective states, Table 1$)$. The decrease in rate constants with increasing temperature suggests that $H / D$ exchange between the protein and $\mathrm{D}_{2} \mathrm{O}$ involves a long-lived reaction complex that is unfavorable at high temperatures. This type of behavior has been reported previously in smaller oligomer systems [16], with other examples of this behavior to be found in [45].

\section{Maximum H/D Exchange Levels as a Function of Temperature}

Figure 6 shows a plot of the maximum H/D exchange level as a function of temperature for the +5 and +9 states of cytochrome $c$. From 300 to $\sim 335 \mathrm{~K}$, the exchange levels increase slightly: $53 \rightarrow 61$ for the +5 state; and, $63 \rightarrow 69$ for the +9 state. Between $\sim 335$ and $\sim 385$ $\mathrm{K}, \sim 60$ new sites exchange for the +5 state $(61 \rightarrow 120)$, while only $\sim 40$ new sites $(69 \rightarrow 109)$ exchange for the +9 state. The exchange levels cross over this temperature range such that the compact +5 state exchanges at a higher level than the elongated +9 state. McLafferty and coworkers [8] (and Valentine et. al.) [11] have previously argued that conformers with compact tertiary structures may undergo lower levels of exchange than open tertiary forms because some hydrogens are protected on interior sites: an argument that is based on what is known about H/D exchange of proteins in 


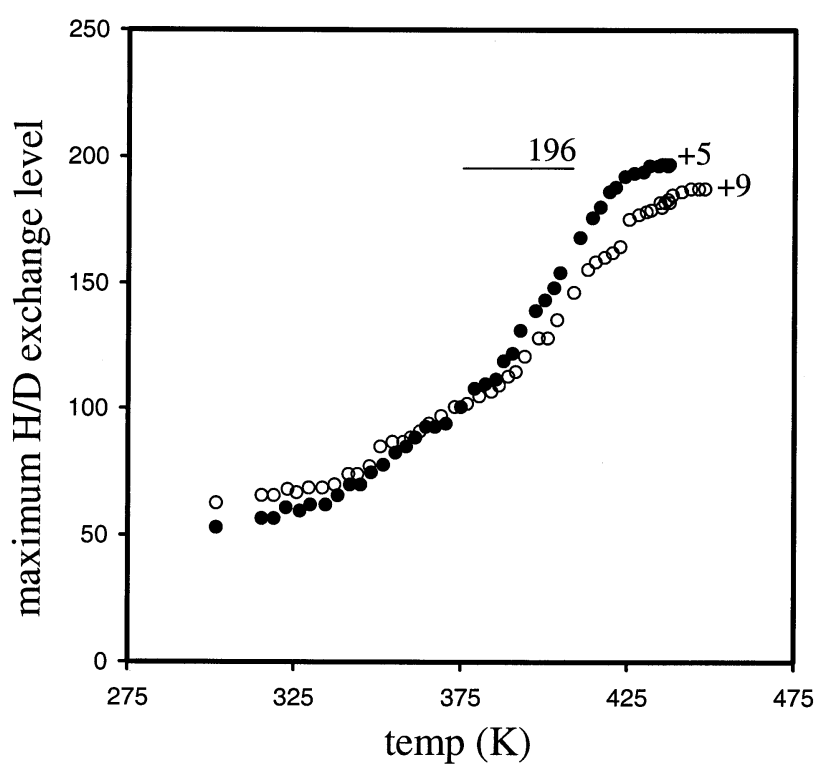

Figure 6. Maximum H/D exchange levels for the +5 (solid circles) and +9 (open circles) charge states of cytochrome $c$ as a function of temperature. The horizontal line shows the 196 level associated with the total number of exchange sites for a nonprotonated cytochrome $c$ in the gas phase. (See text for discussion.) From multiple temperature dependent data sets we estimate uncertainties $(1 \sigma)$ of \pm 3 and \pm 6 hydrogens for the +5 and +9 charge states, respectively at each temperature.

solution $[46,47]$. Although this may be the case at room temperature, it clearly is not true at high temperatures; the compact +5 state exchanges more completely than the elongated +9 conformer at all temperatures above $\sim 380 \mathrm{~K}$. Thus, these results appear to highlight the importance of secondary structure or lack of tertiary structure (discussed below) in the protection of sites. We previously invoked a similar argument to explain the very low levels of exchange that we observed at room temperature for the elongated +8 to +18 states [11].

Above $385 \mathrm{~K}$, the maximum exchange levels of both conformers increase at similar rates. The +5 state reaches a maximum level of $\sim 200$ (the maximum $196+$ $4 \mathrm{H}$ possible for this state) near $\sim 430 \mathrm{~K}$. The number of hydrogens exchanged for the +9 state begins to level off near this temperature; but it does so at a value of $\sim 190$ : 14 below the $204(196+8 \mathrm{H})$ maximum level for this charge state.

\section{Molecular Modeling Simulations: A Stationary Charge Site Model}

The observation that the compact $(+5)$ state exchanges more completely at high temperatures than does the elongated $(+9)$ state is somewhat surprising. In order to understand this result in more detail we have carried out molecular modeling simulations of compact $(+5)$ and elongated $(+9)$ conformers as a function of temperature. The results of these simulations are summarized by estimating the number of exchangeable sites that fall within a specified distance of the protonation site (as discussed above). Overall, the results that are shown below are in poor quantitative agreement with experiment; however, the results do provide a qualitative picture that is valuable.

Our initial model determined the number of exchangeable sites using a model where the charges are fixed to specific basic residues. We refer to this as the stationary charge model. The non-heme charge sites used in these calculations were assigned to residues Lys-5, Lys-25, Lys-39, and Lys-73 [for the compact (+5) state] and residues Lys-5, Lys-27, Arg-38, Lys-55, Lys72, Lys-79, Lys-99, and Lys-100 [for the elongated (+9) state]. The heme iron atom cannot shuttle a deuterium onto the protein via the proposed relay mechanism; however it is possible that a deuterium can be transferred to the negatively charged heme carboxylate groups with concomitant proton abstraction from a neighboring site. While we believe this is probably not a primary mechanism for exchange, we include this possibility because in some simulations we observe interactions of one or both carboxylate groups with other regions of the protein. This is more frequent for the compact structure.

Figure 7 shows the number of exchangeable sites that come within the $5 \AA$ radius of at least one protonation site (or charged carboxylate group) as a function of time at 300,400 and $500 \mathrm{~K}$. Simulations for both conformers show that the number of exchangeable sites that become accessible abruptly increases at short times and then begins to level off. This behavior can be understood by considering that at short times those exchangeable sites that are near the protonation site become accessible when small structural changes occur; large structural changes that allow exchangeable sites that are more remotely situated with respect to the protonation site require longer times to become accessible.

Several other aspects of the simulations are also notable. For example, both conformer types exhibit greater levels of exchange as the temperature is increased-a result that reflects the increase in motion that occurs as temperature is increased. Finally, we note that the model also captures the qualitative changes in the exchange level that are observed experimentally for compact and elongated states. At 500 ps, the $300 \mathrm{~K}$ simulation shows that the elongated structure exchanges more sites (86) than the compact state (61); however, at $500 \mathrm{~K}$, the 500 ps exchange level of the compact state (111) exceeds that of the elongated structure (98).

Additional insight can be obtained by examining the structures obtained from the simulations. Figure 8 shows illustrations of structures obtained at 5, 200, and 500 ps for the dynamics simulation of the compact structure at 300 and $500 \mathrm{~K}$. The locations of exchangeable sites are designated by small spheres. At 5 ps, all of the charge sites as well as a few exchangeable hydrogens that are close to the charge sites are accessible in both the 300 and $500 \mathrm{~K}$ simulations. At longer times (100 


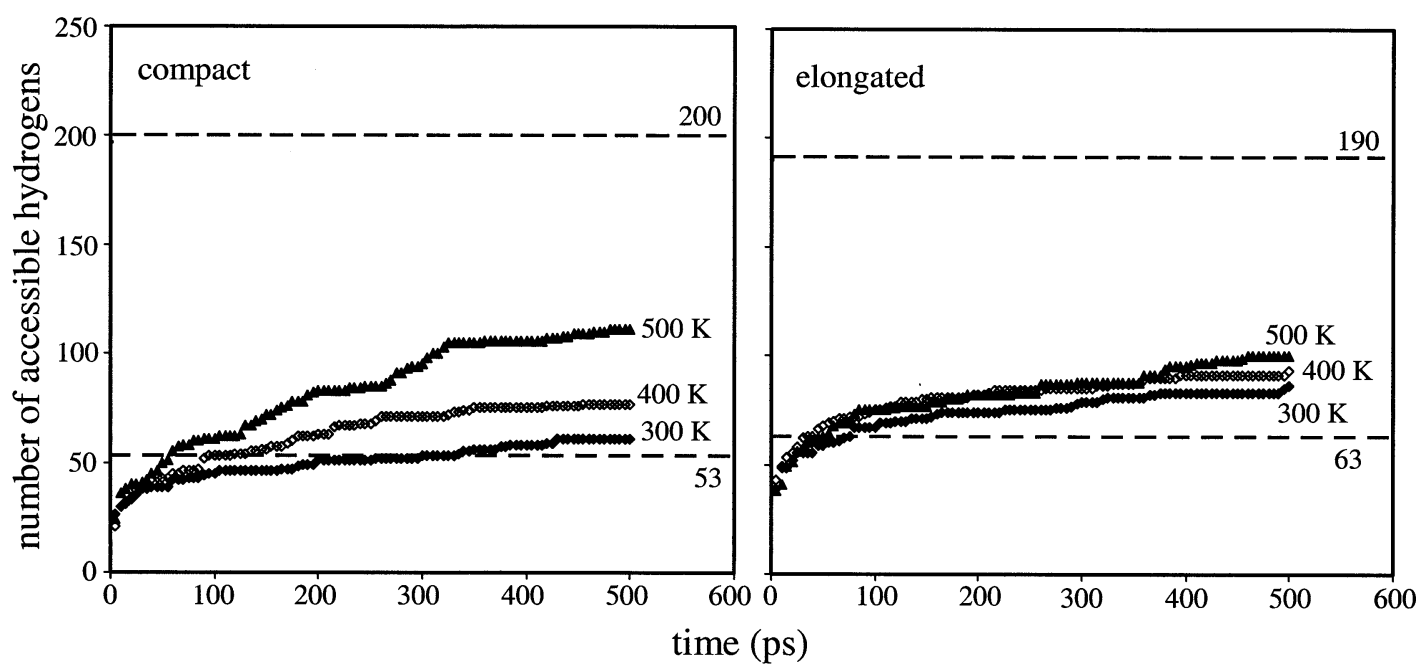

Figure 7. The plot on the left shows the number of accessible hydrogens as a function of time calculated from the molecular dynamics simulations of the compact structure at 300, 400, and $500 \mathrm{~K}$. The plot on the right shows the number of accessible hydrogens as a function of time calculated from the molecular dynamics simulations of the elongated structure at the same temperatures. The dashed lines represent the maximum experimental exchange levels at $\sim 300$ and $440 \mathrm{~K}$ for the compact $(+5)$ conformer and the elongated (+9) conformer.

$300 \mathrm{~K}$
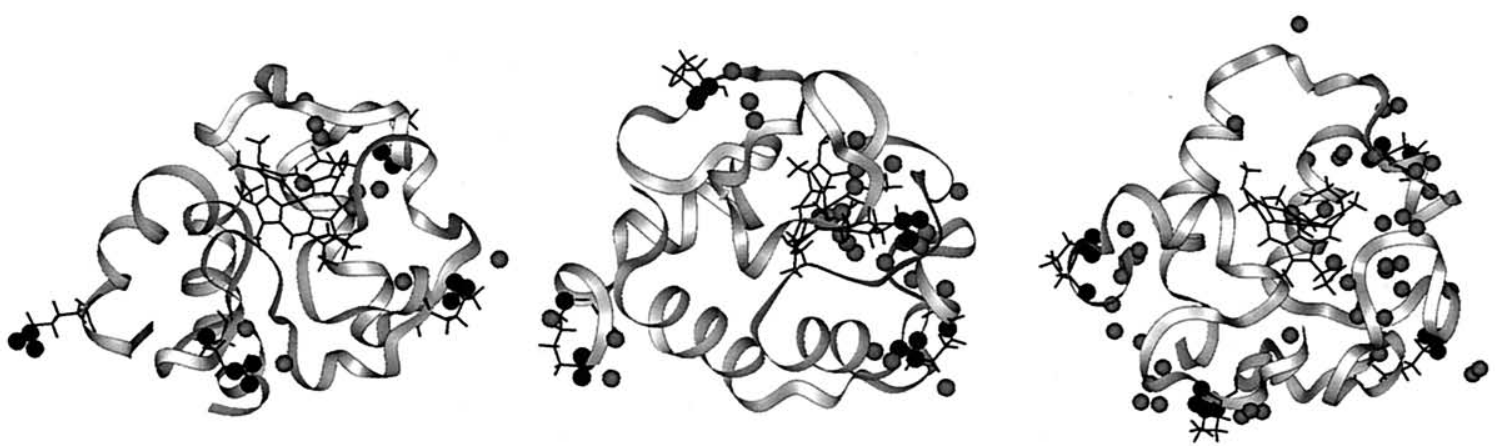

$500 \mathrm{~K}$
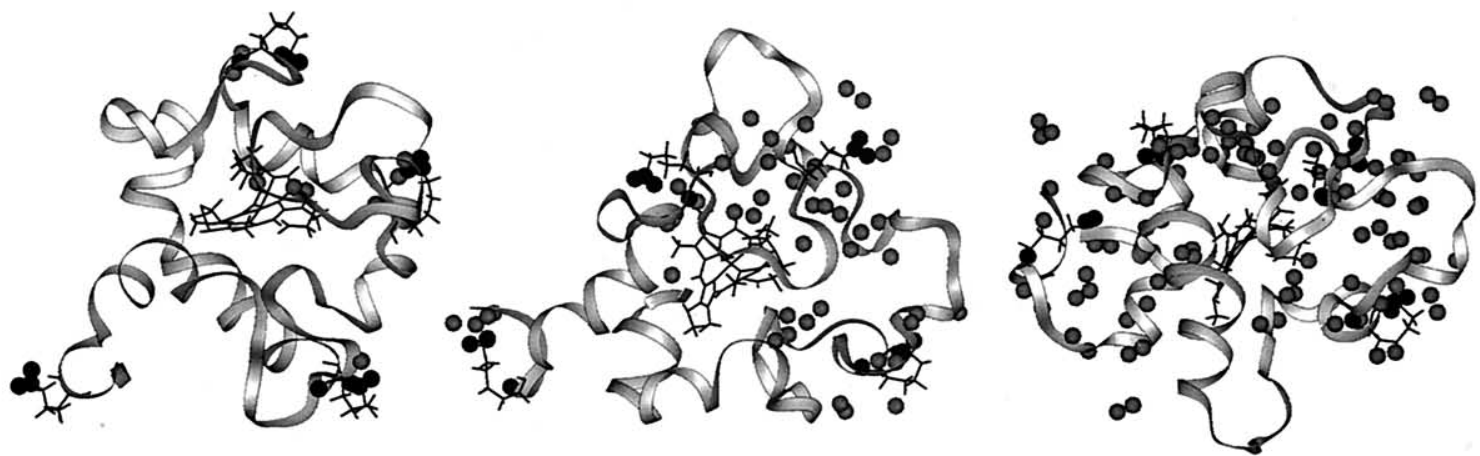

\section{5 ps}

$100 \mathrm{ps}$

$500 \mathrm{ps}$

Figure 8. Ribbon structures obtained at 5,100, and $500 \mathrm{ps}$ in the dynamics simulation of the compact structure at $300 \mathrm{~K}$ (top row of structures) and $500 \mathrm{~K}$ (bottom row). Gray circles represent calculated accessible hydrogens. Charge sites obtained from Coulomb energy calculations (see text) and used in the dynamics simulations, are shown as stick representations and were assumed to be stationary for this calculation. Black circles represent accessible hydrogens on protonated residues. 

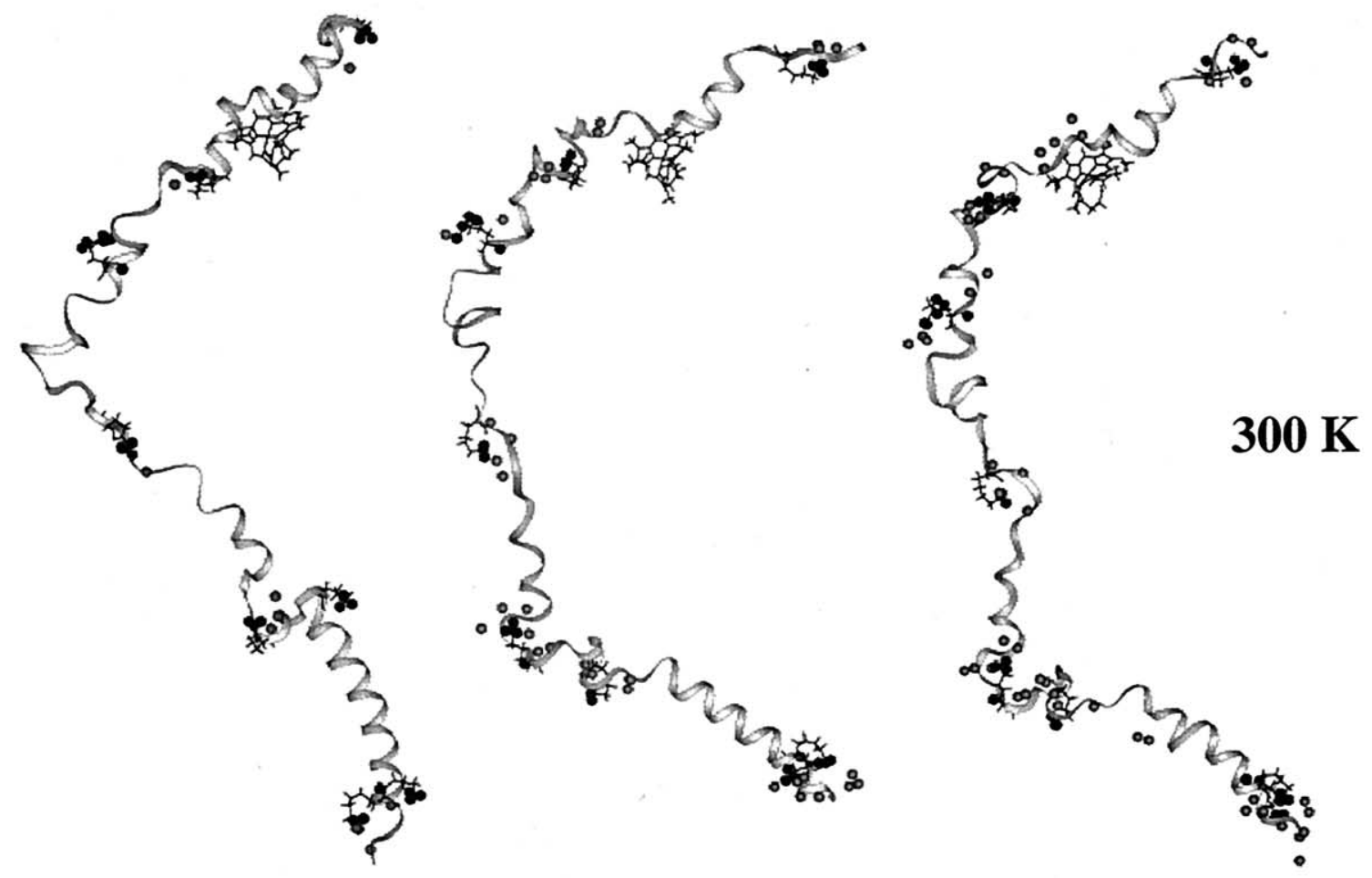

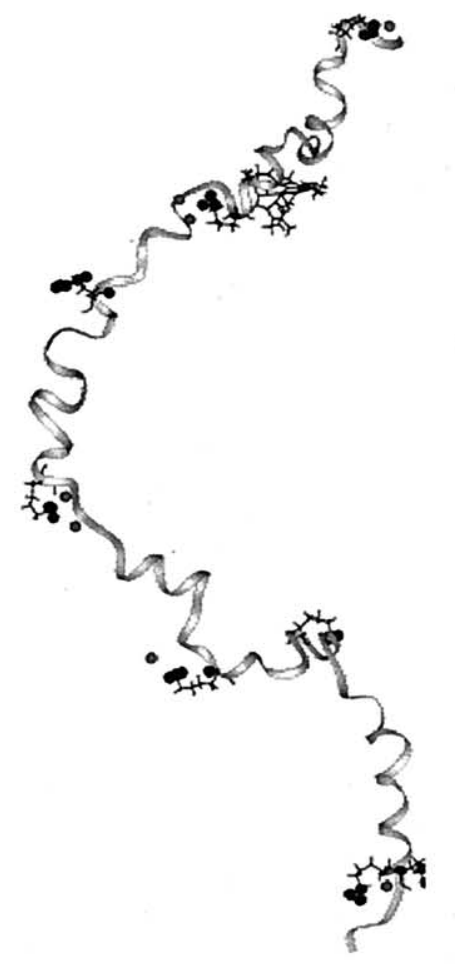

5 ps

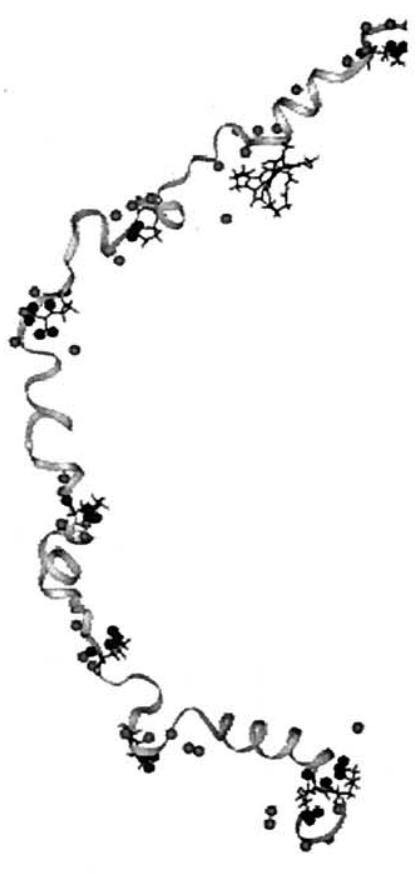

$100 \mathrm{ps}$

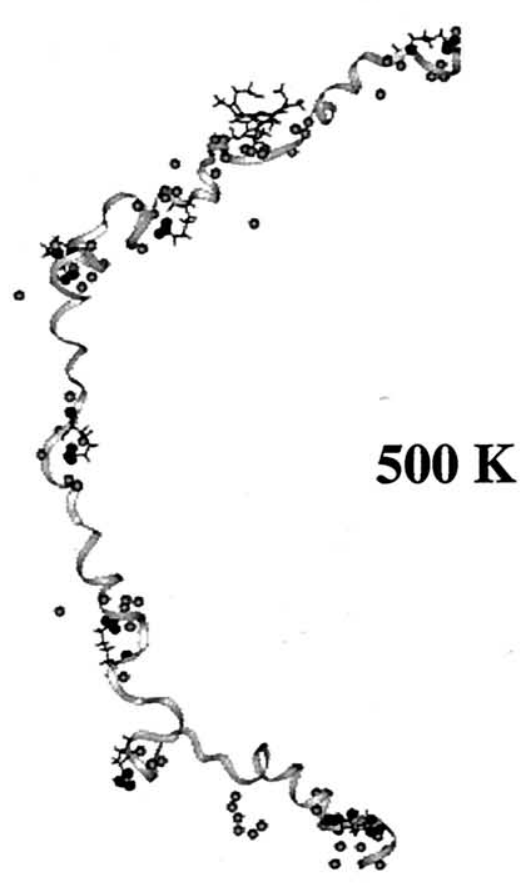

500 ps

Figure 9. Ribbon structures obtained in the dynamics simulation of the elongated structure at 300 and $500 \mathrm{~K}$ (see Figure 8).

and 500 ps), several exchangeable hydrogens that are more distant to the charge sites are observed to have become accessible. It is clear that temperature has a dramatic effect on the total exchange levels that are observed. Those sites that have not exchanged exist primarily along turns that protrude away from the 
center of the molecule; these regions do not come into contact with the charge sites and thus do not exchange during the simulation.

Structures for the elongated geometry are shown in Figure 9. As observed in the simulations of the compact conformer, at short simulation times (5 ps), the exchangeable sites are localized around the sites of protonation. Here, the open geometry (and greater number of protonation sites) leads to a larger number of accessible sites at $300 \mathrm{~K}$ than is accessible for the compact $(+5)$ state. At high temperatures (and 100 to $500 \mathrm{ps}$ simulation times), large regions of heteratoms between the protonation sites remain unexchanged. This behavior is fairly different from the results observed for the compact conformer. The lack of tertiary folds in the extended confomer prohibits exchange across different regions of the sequence. Because of the relatively few number of times that the elongated structure folds up during the simulations, there is relatively little exchange in regions of the sequence that are distant from the protonation sites.

\section{Molecular Modeling Simulations: A Mobile Charge Site Mode}

The lack of quantitative agreement between the stationary charge model and the experiment suggests that a more sophisticated treatment is necessary. (For a discussion of the mobile proton model and references to groups contributing to the development of this model, see [48].) We have considered several other possibilities. For example the critical distances of 3 and $4 \AA$ were also used; however, the results do not vary substantially from those discussed above. Another reasonable possibility is that the protonation sites may become mobile during the exchange process. If we allow the sites to be mobile at all temperatures, ${ }^{\text {III II }}$ then the model over estimates the exchange levels observed experimentally at low temperatures. Additionally, it does not capture the cross over of the maximum exchange levels of the compact and elongated states as temperature is increased. However, this type of model does pick up the extensive exchange that is observed at high temperatures. Work on a model that incorporates a barrier to transferring protons between basic residues is ongoing in our laboratory.

\section{Summary and Conclusions}

Combined ion mobility and H/D exchange studies of compact (+5) and elongated (+9) conformations of cytochrome $c$ over a temperature range of 300 to $440 \mathrm{~K}$ were reported. The cross sections for these ions are

\footnotetext{
IIII In this model, proton mobility is introduced by examining the distance between protonated and other basic residues on the archived structures and then reconfiguring the charge distribution when non-protonated basic groups are within $\sim 10 \AA$. While this model is at a preliminary stage of development, it shows that the number of exchange positions exceeds the experimental value at $300 \mathrm{~K}$.
}

invariant at all temperatures. However, over this temperature range, the $\mathrm{H} / \mathrm{D}$ exchange levels vary from 53 to 200 for the +5 state; and 63 to 190 for the +9 state. Molecular modeling simulations have been used to examine a simple model for exchange that considers exchange to be possible if a heteroatom site falls within a 5 A distance of a protonation site. This model captures the qualitative experimental findings. It appears that the compact state can exchange at a greater level at high temperatures because the folded structure brings many regions of the polypeptide chain into contact with the charged sites; on the other hand, while the elongated structure is highly accessible to solvent molecules, large regions of the sequence remain distant from protonation sites-even at high temperatures and long simulation times.

\section{Acknowledgments}

The authors thank Yansheng Liu for assistance provided in data collection. They are also grateful to F. W. McLafferty, M. F. Jarrold, and Anne E. Counterman for many stimulating discussions and preprints of recent work. This work was supported by a grant from the NSF (Grant No. CHE-0078737).

\section{References}

1. Pauling, L.; Corey, R. B.; Branson, H. R. Proc. Natl. Acad Sci. USA 1951, 37, 205.

2. Hoaglund-Hyzer, C. S.; Counterman, A. E.; Clemmer, D. E. Chem. Rev. 1999, 99, 3037.

3. Desai, R. R.; Osterhout, J. J.; Klibanov, A. M. J Am. Chem. Soc. 1994, 116, 9420.

4. (a) Careri, G.; Gratton, E.; Yang, P. H.; Rupley, J. A. Nature 1980, 284. (b) Poole, P. L.; Finney, J. L. Biopolymers 1983, 22, 255. (c) Schinkel, J. E.; Downer, N. W.; Rupley, J. A. Biochemistry 1985, 24, 352. (d) Rupley, J. A.; Careri, G. Adv. Protein Chem. 1991, 41, 37. (e) Prestrelski, S. J.; Tedeschi, N.; Arakawa, T.; Carpenter, J. F. Biophys. J. 1993, 65, 661.

5. Fenn, J. B.; Mann, M.; Meng, C. K.; Wong, S. F.; Whitehouse, C. M. Science 1989, 246, 64.

6. Karas, M.; Hillenkamp, F. Anal. Chem. 1988, 60, 2299.

7. Winger, B. E.; Light-Wahl, J. J.; Rockwood, A. L.; Smith, R. D. J Am. Chem. Soc. 1992, 114, 5897.

8. Suckau, D.; Shi, Y.; Beu, S. C.; Senko, M. W.; Quinn, J. P.; Wampler, F. M., III; McLafferty, F. W. Proc. Natl. Acad Sci. USA 1993, 90, 790

9. Wood, T. D.; Chorush, R. A.; Wampler, F. M., III; Little, D. P.; O'Connor, P. B.; McLafferty, F. W. Proc. Natl. Acad Sci. USA 1995, 92, 2451.

10. Cassady, C. J.; Carr, S. R. J. Mass Spectrom. 1996, 31, 247.

11. Valentine, S. J.; Clemmer, D. E. J Am. Chem. Soc. 1997, 119, 3558.

12. McLafferty, F. W.; Guan, Z.; Haupts, U.; Wood, T.; Kelleher, N. L. J. Am. Chem. Soc. 1998, 120, 4732.

13. Green, M. K.; Lebrilla, C. B. Mass Spectrom. Rev. 1997, 16, 53.

14. Freitas, M. A.; Hendrickson, C. L.; Emmett, M. R.; Marshall, A. G. Int. J. Mass Spectrom. 1999, 185/186/187, 565.

15. Gard, E.; Green, M. K.; Bregar, J.; Lebrilla, C. B. J. Am. Soc. Mass Spectrom. 1994, 5, 623.

16. Campbell, S.; Rodgers, M. T.; Marzluff, E. M.; Beauchamp, J. L. J. Am. Chem. Soc. 1995, 117, 12840.

17. Gur, E. H.; de Koning, L. J.; Nibbering, N. M. M. J. Am. Soc. Mass Spectrom. 1995, 6, 466 
18. (a) Freitas, M. A.; Hendrickson, C. L.; Emmett, M. R.; Marshall, A. G. J. Am. Soc. Mass Spectrom. 1998, 9, 1012. (b) Freitas, M. A.; Shi, S. D.-H.; Hendrickson, C. L.; Marshall, A. G. J. Am. Chem. Soc. 1998, 120, 10187. (c) Freitas, M. A.; Marshall, A. G. Int. J. Mass Spectrom. 1999, 182/183, 221.

19. Wyttenbach, T.; Bowers, M. T. J. Am. Soc. Mass Spectrom. 1999, 10,9 .

20. (a) Schnier, P. F.; Gross, D. S.; Williams, E. R. J. Am. Chem. Soc. 1995, 117, 6747. (b) Gross, D. S.; Schnier, P. D.; RodriguezCruz, S. E.; Fagerquist, C. K.; Williams, E. R. Proc. Natl. Acad. Sci. USA 1996, 93, 3143. (c) Williams, E. R. J. Mass Spectrom. 1996, 31, 831.

21. (a) Cassady, C. J.; Wronka, J.; Kruppa, G. H.; Laukien, F. H. Rapid Commun. Mass Spectrom. 1994, 8, 94. (b) Zhang, X.; Cassady, C. J. J. Am. Soc. Mass Spectrom. 1996, 7, 1211.

22. (a) Valentine, S. J.; Anderson, J. G.; Ellington, A. E.; Clemmer, D. E. J. Phys. Chem. B 1997, 101, 3891. (b) Valentine, S. J.; Counterman, A. E.; Clemmer, D. E. J. Am. Soc. Mass Spectrom. 1997, 8, 954 .

23. McLuckey, S. A.; Stephenson, J. R., Jr. Anal. Chem. 1997, 69, 281.

24. (a) Kaltashov, I. A.; Fenselau, C. C. J. Am. Chem. Soc. 1995, 117, 9906. (b) Adams, J.; Strobel, F.; Reiter, A. J. Am. Soc. Mass Spectrom. 1996, 7, 30. (c) Kaltashov, I. A.; Fenselau, C. C. Proteins 1997, 27, 165.

25. (a) Covey, T. R.; Douglas, D. J. J. Am. Soc. Mass Spectrom. 1993, 4, 616. (b) Collings, B. A.; Douglas, D. J. J. Am. Chem. Soc. 1996, $118,4488$.

26. Cox, K. A.; Julian, R. K.; Cooks, R. G.; Kaiser, R. E. J. Am. Soc. Mass Spectrom. 1994, 5, 127.

27. (a) von Helden, G.; Wyttenbach, T.; Bowers, M. T. Science 1995, 267, 1483. (b) Wyttenbach, T.; von Helden, G.; Bowers, M. T. J. Am. Chem. Soc. 1996, 118, 8355.

28. (a) Clemmer, D. E.; Hudgins, R. R.; Jarrold, M. F. J. Am. Chem. Soc. 1995, 117, 10141. (b) Shelimov, K. B.; Jarrold, M. F. J. Am. Chem. Soc. 1996, 118, 10313. (c) Shelimov, K. B.; Clemmer, D. E.; Hudgins, R. R.; Jarrold, M. F. J. Am. Chem. Soc. 1997, 119, 2240. (d) Woenckhaus, J.; Mao, Y.; Jarrold, M. F. J. Phys. Chem. B. 1997, 101, 847.

29. Liu, Y.; Clemmer, D. E. Anal. Chem. 1997, 69, 2504.

30. (a) Hagen, D. F. Anal. Chem. 1979, 51, 870. (b) St. Louis, R. H.; Hill, H. H. Crit. Rev. Anal. Chem. 1990, 21, 321. (c) von Helden, G.; Hsu, M. T.; Kemper, P. R.; Bowers, M. T. J. Chem. Phys. 1991, 95, 3835. (d) Jarrold, M. F. J. Phys. Chem. 1995, 99, 11. (e) Clemmer, D. E.; Jarrold, M. F. J. Mass Spectrom. 1997, 32, 577. (f) Liu, Y.; Valentine, S. J.; Counterman, A. E.; Hoaglund, C. S.; Clemmer, D. E. Anal. Chem. 1997, 69, 728A.

31. Hoaglund, C. S.; Liu, Y.; Ellington, A. D.; Pagel, M.; Clemmer, D. E. J. Am. Chem. Soc. 1997, 119, 9051.

32. Horn, D. M.; Breuker, K.; Frank, A. J.; McLafferty, F. W. J. Am. Chem. Soc. 2001, 123, 9792.

33. (a) Reimann, C. T.; Velazquez, I; Tapia, O. J. Phys Chem. B 1998, 102, 9344. (b) Arteca, G. A.; Velazquez, I.; Reimann, C. T.; Tapia, O. J. Chem. Phys. 1999, 111, 4774. (c) Velazquez, I.; Reimann, C. T.; Tapia, O. J. Am Chem. Soc. 1999, 121, 11468.

34. (a) Hudgins, R. R.; Mao, Y.; Ratner, M. A.; Jarrold, M. F. Biophys. J. 1999, 76, 1591. (b) Mao, Y.; Woenckhaus, J.; Kolafa, J.; Ratner, M. A.; Jarrold, M. F. J. Am. Chem. Soc. 1999, 121, 2712. (c) Hudgins, R. R.; Jarrold, M. F. J. Am. Chem. Soc. 1999, 121, 3494. (d) Mao, Y.; Ratner, M. A.; Jarrold, M. F. J. Phys. Chem. B 1999, 103, 10017.

35. Counterman, A. E.; Clemmer, D. E. J. Am. Chem. Soc. 1999, 121, 4031.

36. Mao, Y.; Woenckhaus, J.; Kolafa, J.; Ratner, M. A.; Jarrold, M. F. J. Am. Chem. Soc. 1999, 121, 2712.

37. Mao, Y; Ratner, M. A.; Jarrold, M. F. J. Phys. Chem. B. 1999, 103, 10017.

38. Mason, E. A.; McDaniel, E. W. Transport Properties of Ions in Gasses. Wiley: New York, 1988.

39. Williams, E. R. J. Mass Spectrom. 1996, 31, 831.

40. He, F.; Hendrickson, C. L.; Marshall, A. G. J. Am. Soc. Mass Spectrom. 2000, 11, 120.

41. Cheng, X.; Fenselau, C. Int. J. Mass Spectrom Ion Processes 1992, 122, 109.

42. (a) Gard, E.; Green, M. K.; Bregar, J.; Lebrilla, C. B. J. Am. Soc. Mass Spectrom. 1994, 5, 623. (b) Green, M. K.; Lebrilla, C. B. Int. J. Mass Spectrom. Ion Processes 1998, 175, 15.

43. Shvartsburg, A. A.; Jarrold, M. F. Chem Phys Lett 1996, 261, 86.

44. (a) Mesleh, M. F.; Hunter, J. M.; Shvartsburg, A. A.; Schatz, G. C.; Jarrold, M. F. J. Phys. Chem. 1996, 100, 16082. (b) Wyttenbach, T.; von Helden, G.; Batka, J., Jr.; Carlat, D.; Bowers, M. T. J. Am. Soc. Mass Spectrom. 1997, 8, 275.

45. Blint, R. J.; McMahon, T. B.; Beauchamp, J. L. J. Am. Chem. Soc. 1974, 96, 1269.

46. Wand, A. J.; Roder, H.; Englander, S. W. Biochemistry 1996, 35, 1107.

47. Jeng, F. F.; Englander, S. W.; Elove, G. A.; Wand, A. G.; Roder, H. Biochemistry 1990, 29, 10433.

48. (a) Dongré, A. R.; Jones, J. L.; Somogyi, A.; Wysocki, V. H. J. Am. Chem. Soc. 1996, 118, 8365. (b) Wysocki, V. H.; Tsaprailis, G.; Smith, L. L.; Breci, L. A. J. Mass Spectrom. 2000, 35, 1399. 